\title{
Effect of Kaleidoscope on Pain Perception of Children Aged 4-6 Years During Intravenous Cannulation
}

\author{
Dipeesh Kunjumon ${ }^{1}$, Vinil Upendrababu ${ }^{2}$ \\ ${ }^{1}$ Department of Nursing, North West Anglia NHS Foundation Trust, Peterborough, United Kingdom \\ ${ }^{2}$ Department of Nursing, College of Health Sciences, Wollega University, Nekemte, Ethiopia
}

Email address:

vinilupendrababu@gmail.com (V. Upendrababu)

\section{To cite this article:}

Dipeesh Kunjumon, Vinil Upendrababu. Effect of Kaleidoscope on Pain Perception of Children Aged 4-6 Years During Intravenous Cannulation. American Journal of Nursing Science. Vol. 7, No. 4, 2018, pp. 137-142.doi: 10.11648/j.ajns.20180704.14

Received: May 14, 2018; Accepted: May 30, 2018; Published: June 25, 2018

\begin{abstract}
Management of children's health includes medical procedures that may be painful or stressful to the child. Yet, despite extensive research findings, policy guidelines and practice, standard recommendations for optimal management of pediatric pain, clinical practices remain inadequate. Hence the investigator conducted a study to assess the effect of distraction method like kaleidoscope in managing pain in children during procedure like intravenous cannulation. The main objective of this study was to assess the effect of kaleidoscope on pain perception of children aged $4-6$ years during intravenous cannulation. The research approach adopted for this study was true experimental and the design was post test only control group design. The study was conducted in Upasana hospital, Kollam among 30 children aged $4-6$ years who were admitted in the pediatric ward, with 15 children each in experimental and control group. After obtaining consent from caregivers, demographic data was collected and physiological parameters like heart rate and $\mathrm{SPO}_{2}$ were measured using pulse oximeter, five minutes prior to the cannulation. The children in the experimental group were introduced to kaleidoscope before the cannulation and were told to look through it during the procedure. During cannulation, the objective pain was assessed by using FLACC scale and the physiological parameters were again measured. Five minutes after the procedure, the children were asked to explain the pain during cannulation using Wong Baker Faces Pain Rating scale. The mean pain scores of experimental group was significantly less than that of the control group $(\mathrm{p}<0.05)$. There was a significant relationship between pain scores and variability in heart rate ( $\mathrm{r}=0.93$ according to FLACC scale \& $\mathrm{r}=0.85$ according to WBFPRS) and $\mathrm{SPO}_{2}(\mathrm{r}=0.93$ according to FLACC scale \& $\mathrm{r}=0.86$ according to WBFPRS) of children during intravenous cannulation. To sum up, the kaleidoscope was shown to be effective in managing pain in children aged 4-6 years, during intravenous cannulation. So distraction can be used effectively in pain management of children.
\end{abstract}

Keywords: Effect, Kaleidoscope, Pain, Perception, Children Aged 4-6 Years, Intravenous Cannulation

\section{Introduction}

During hospitalization, children may undergo a wide range of interventions, many of which can be traumatic, stressful and painful. Despite advances in both pharmacological and non-pharmacological methods of preventing and treating pain, many children still endure unacceptable levels of pain and distress during their hospitalization. The procedure made by a syringe in children are the most common source of pain and venepuncture has been evaluated as one of the most painful interventions commonly applied to children. Empirical evidence shows that unrelieved pain in children has only harmful consequences, with no benefits.

Health care professionals in hospital settings have a responsibility to reduce pain and anxiety as much as possible, while maintaining patient safety [1]. Optimal pain management is the right of all patients and the responsibility of all health professionals. Thus to reduce the emotional and physical effects of painful interventions and to prevent long term results of pain in children, the nurses should be able to manage the painful procedures. Among the pharmacological and non-pharmacological interventions (independent or complementary), non- pharmacological methods have been considered to be favorable strategies for pain management and research focusing on nurses use of non-pharmacological 
methods for relief of children's pain has increased in recent years. One of the effective non-pharmacological methods is distraction.

The study titled "Psychological intervention for needle related procedural pain and distress in children and adolescents" done in July 2006, depicts that psychological interventions are effective in reducing needle related pain and distress in children and adolescents. The research was conducted as 28 trials with 1951 participants. The interventions used were distraction, combined cognitivebehavioral interventions and hypnosis.

Lander M, Hodgins (2011) conducted study on Children's coping with venipuncture. Children's strategies for coping with the pain and distress of venipuncture were examined in this descriptive study. Eighty-five children (aged 5-13 years) were interviewed prior to and following blood collection. Twenty-seven different strategies were identified from the children's responses. active involvement in procedure, behaviour-regulating cognitions, cognitive reappraisal, direct efforts to maintain control, diversionary thinking, emotionregulating cognitions, information seeking, reality-oriented working through, reliance on health-care interventions, support seeking and avoidance and catastrophizing.

Distraction work best for short, intense pain lasting a few minute such as during an invasive procedure or while waiting for an analgesic to work. The reticular activating stimuli inhibit painful stimuli if a person receives sufficient of excessive sensory input. With meaningful sensory stimuli, a person can ignore or become unaware of pain [2].

In a study which is conducted by Himali Raj Prajapathi in a selected hospital in Ahamadabad, proved that kaleidoscope is one of the effective strategy to reduce the physical stress during venipuncture procedure among hospitalized preschooler children [9]. In addition to, another study which is conducted on selected hospitals of Coimbatore districts of tamilnadu had also shown the significance of using kaleidoscope among children while doing the painful procedures to divert their attention [10]. In these both studies there is a significant changes in the post test pain scale score ofexperimental group had been identified after the implementation of kaleidoscope during the invasive procedures. Moreover, Becky Hix in her study which is conducted in university of canetrbury also signifies the importance of using different distraction methods while doing the invasive procedures on children [11].

One of the method of distraction, kaleidoscope is found to be effective in reducing pain in children during venepuncture [3]. Despite the plethora of studies, nonpharmacological pain reduction remedies such as the proven methods have not been effectively used for routine painful procedures for children in clinical practice. Hence the investigator felt the need to conduct a study related to use of kaleidoscope, one of the distraction methods, in pain management of children during intravenous cannulation.

\subsection{Statement of the Problem}

The statement of problem for the present study was"Effect of kaleidoscope on pain perception during intravenous cannulation among children aged 4-6 years, admitted in Upasana hospital, Kollam.”

\subsection{Objectives}

The objectives of this study were

1. To compare the pain perception scores of experimental and control group during intravenous cannulation.

2. To find the association of pain perception scores of experimental group with the selected demographic variables such as age, sex and previous experience of cannulation in children.

\subsection{Hypotheses}

In this study, the hypotheses were as follows.

$\mathrm{H}_{1}$ : The mean pain scores of experimental group will be significantly less than that of the control group.

$\mathrm{H}_{2}$ : There will be significant association between pain perception scores of experimental group and selected demographic variables such as age, sex and previous experience of children.

\section{Method}

\subsection{Design and Setting}

The research approach adopted for this study was true experimental and the design was post test only control group design. The design can be diagrammatically represented as

Experimental group- R X O

Control group - $\mathrm{RO}$ where $\mathrm{R}$ is randomization, $\mathrm{O}$ is assessment of pain. $\mathrm{X}$ is treatment (use of kaleidoscope). In this study, the dependent variable was pain perception of children aged 4-6 years and the independent variable was effect of kaleidoscope. The study was conducted in Pediatric ward of Upasana hospital, Kollam.

\subsection{Sample Size and Sampling Technique}

In this study, a sample of 30 children of age group 4-6 years were included, who were divided into experimental and control groups with 15 sample in each group. Simple random sampling technique was adopted for assigning sample in each group, which is a basic probability sampling involving the selection of sample members from a sample frame through completely random procedures.

\subsection{Tool and Technique}

\subsubsection{Tool}

A tool to assess the effect of kaleidoscope on pain perception of preschoolers during intravenous cannulation includes the following.

Section A: Demographic data of child which involves age, sex, birth order, type of family, previous experience ofcannulation, site of cannulation, size of cannula used and number of cannulation in the present hospitalization.

Section B: Assessment of pain during intravenous 
cannulation. Standardised tools were used to asses the pain.

Part I: Flacc scale to assess objective pain of the child.
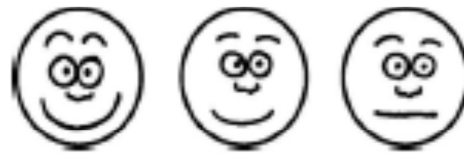

2

4

Part II: Wong Baker's Faces Pain Rating scale to obtain subjective pain of child.

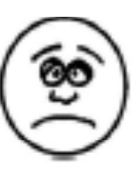

6

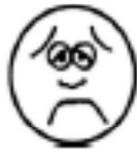

8

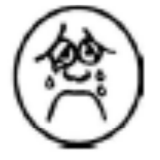

10

Figure 1. Wong Baker Faces Pain Rating scale.

The score represent the following [4]

$0=$ very happy, no pain.

$2=$ hurts just a little bit.

$4=$ hurts a little more

$6=$ hurts even more.
$8=$ hurts a whole lot.

$10=$ hurts as much as you can imagine.

The FLACC behavioral pain assessment scale is an easily administered tool to assess acute pain in infants and young children.

Table 1. Flacc scale.

\begin{tabular}{|c|c|c|c|}
\hline CATEGORY & SCORE-1 & 2 & 3 \\
\hline Face $(F)$ & No particular expression or smile & $\begin{array}{l}\text { Occasional grimace or frown, withdrawn, } \\
\text { disinterested }\end{array}$ & $\begin{array}{l}\text { Frequent to constant quivering chin, } \\
\text { clenched jaw }\end{array}$ \\
\hline Legs (L) & Normal position or relaxed & Uneasy, restless, tense & Kicking, or legs drawn up \\
\hline Activity (A) & $\begin{array}{l}\text { Lying quietly, normal position, moves } \\
\text { easily }\end{array}$ & Squirming, shifting back and forth, tense & Arched, rigid or jerking \\
\hline Cry (C) & No cry (awake or asleep) & $\begin{array}{l}\text { Moans or whimpers; occasional } \\
\text { complaint }\end{array}$ & $\begin{array}{l}\text { Crying steadily, screams or sobs, } \\
\text { frequent complaints }\end{array}$ \\
\hline Consolability (C) & Content, relaxed & $\begin{array}{l}\text { Reassured by occasional touching, } \\
\text { hugging or being talked to, distractible }\end{array}$ & Difficult to console or comfort \\
\hline
\end{tabular}

Each of the five categories (F) Face; (L) Legs; (A) Activity; (C) Cry; (C) Consolability is scored from 0-2, which results in a total score between zero and ten.

\subsubsection{Technique}

1. Semi structured interview schedule to obtain demographic data of the sample.

2. Observation to assess the pain using FLACC scale

The intensity of pain was classified according to the pain scores as follows.

$0=$ no pain, $1-3=$ mild pain, $4-7=$ moderate pain and $8-10=$ severe pain.

\subsection{Validity and Reliability}

The tool to assess the pain perception of children during intravenous cannulation was validated by seven experts in the respective specialty. The reliability of FLACC scale was assessed by interrater method. Using Karl Pearson's formula for correlation coefficient, $\mathrm{r}=0.76$. Using Spearman Brown's prophecy formula, $\mathrm{R}=0.86$. The tool was found to be reliable.

\subsection{Data Collection Procedure}

After obtaining informed consent, demographic data were collected and explained the use of kaleidoscope to the child. After 5 minutes, intravenous cannulation was done by staff nurse and child was told to look through the kaleidoscope during intravenous cannulation. The investigator assisted the child in holding the kaleidoscope. The investigator assessed the objective pain of the child using FLACC scale. Five minutes after the procedure, the subjective pain was assessed using Wong Bakers Faces Pain Rating Scale, which was reported by children themselves.

\section{Result}

The data thus obtained were analyzed and presented under the following sections.

\subsection{Demographic Data of Sample}

1. Among the total sample, there was an equal distribution of children in all age groups of experimental group, whereas in the control group, $46.67 \%$ of sample belongs to 5 years and the rest belong to 4 years and 6 years with $26.67 \%$ each.

2. Regarding the sex of the children, $60 \%$ of sample in experimental group and $46.67 \%$ of the control group were males.

3. $46.67 \%$ of the experimental group and $40 \%$ of the control group were undergoing cannulation for the first time and the remaining children had previous experience of cannulation.

4. About the number of cannulation in the present hospitalization, $80 \%$ of the experimental group and $60 \%$ of control group children had cannulation for the first time in the present hospitalization.

5. $73.33 \%$ of both experimental and control group belong to birth order of 1 and nearly $30 \%$ of both group belong to birth order of 2 .

6. Nearly $90 \%$ of the sample inexperimental as well as 
control group were from nuclear family.

7. All children had cannulation in the upper limb.

8. The entire sample selected for the study had cannulation with the cannula sized $24 \mathrm{G}$.

\subsection{Intensity of Pain}

\subsubsection{Pain Scores According to Flacc \& Wbfprs of Experimental Group}

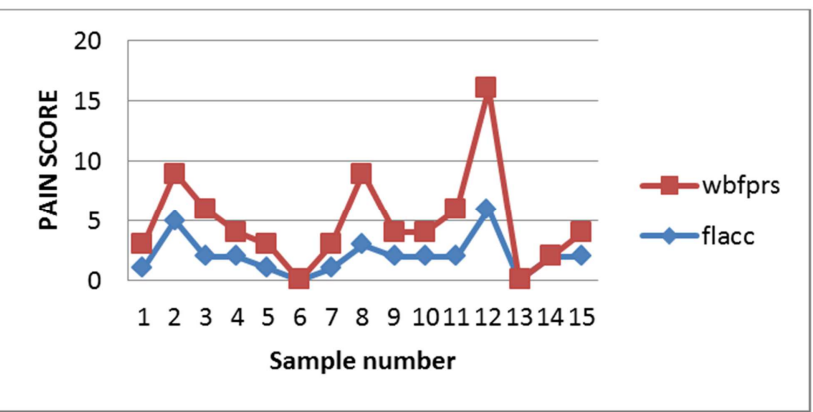

Figure 2. Line diagram showing distribution of FLACC and WBFPRS pain scores of experimental group.

It is clear from the graph that the pain scores of experimental group according to Wong Baker Faces Rating scale exceeds that of FLACC scale.

\subsubsection{Pain Scores According to Flacc \& Wbfprs of Control Group}

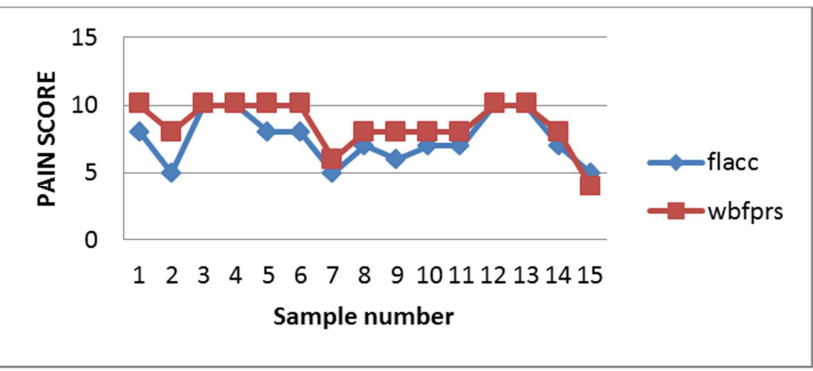

Figure 3. Line diagram showing distribution of FLACC and WBFPRS scores of control group.

It is clear from the figure that the pain scores of control group according to Wong Baker Faces Rating scale exceeds that of FLACC scale.

\subsubsection{Intensity of Pain According to Flacc Scale}

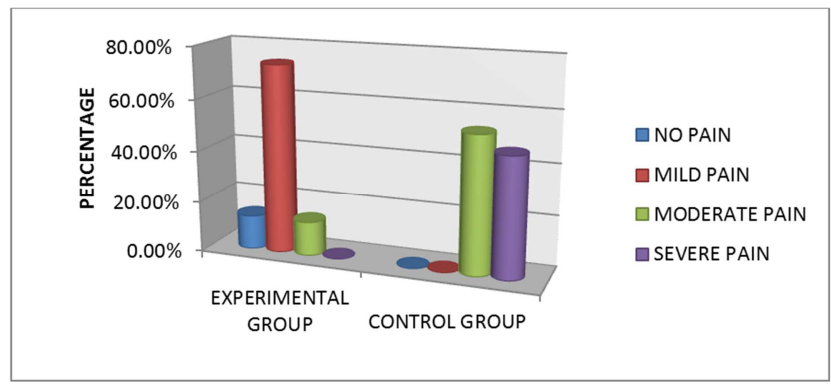

Figure 4. Cylindrical diagram showing distribution of severity of pain according to FLACC scale.
From the above diagram, it is clear that the children in experimental group had mild and moderate pain during cannulation whereas those in the control group had moderate and severe pain, according to FLACC scale.

\subsubsection{Intensity of Pain According to Wong Baker Faces Pain Rating Scale}

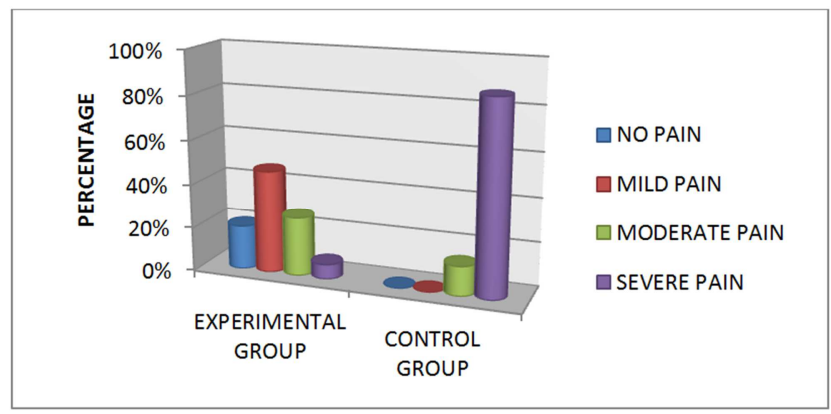

Figure 5. Cylindrical diagram showing distribution of severity of pain according to Wong Baker Faces Pain rating scale.

The above diagram depicts that more than $80 \%$ of the control group had severe pain whereas in experimental group about $45 \%$ had mild pain and about $25 \%$ had moderate pain according to Wong Baker Faces Pain rating scale.

\subsection{Effect of Kaleidoscope on Pain Perception of Children Aged 4-6 Years Undergoing Intravenous Cannulation}

The hypotheses were statistically tested by using 'student $t$ test'.

$\mathrm{H}_{1}$ : The mean pain scores of experimental group will be significantly less than that of the control group.

$\mathrm{H}_{0 \text { : }}$ There will be no difference between the mean pain scores of experimental and control group

Table 2. Mean, standard deviation and t value according to two pain scales.

\begin{tabular}{lllllll}
\hline \multirow{2}{*}{ FLACC } & CATEGORY & $\mathbf{N}$ & Mean & SD & T & P \\
\hline \multirow{2}{*}{ WBFPRS } & Experimental & 15 & 2.1 & 1.6 & \multirow{2}{*}{8.609} & 0.001 \\
& Control & 15 & 7.5 & 1.8 & & \\
& Experimental & 15 & 2.8 & 2.6 & \multirow{2}{*}{7.069} & 0.001 \\
\hline
\end{tabular}

The table above illustrates that according to FLACC scale, the mean pain score of experimental group was 2.1 whereas in control group, it was 7.5. And according to Wong Baker Faces Pain rating scale, in experimental group, it was 2.8 and that in control group was 8.5. The standard deviation in experimental group was 1.6, according to FLACC scaleand that in control group was 1.8. Since the test is statistically significant at $\mathrm{p}=0.001$, according to both scales, $\mathrm{H}_{1}$ is accepted and $\mathrm{H}_{0}$ is rejected.

That is, the mean pain scores of experimental group was significantly less than that of the control group. It indicates that the kaleidoscope was effective in reducing pain perception in children aged 4-6 years, during intravenous cannulation. 


\subsection{Association of Pain Score with Selected Demographic Variables}

1. There was no association found between pain score according to FLACC scale and age of experimental group $(p>0.05)\left(\chi^{2}=2.182 d f=4 p=0.702\right)$

2 . There was no association found between pain score according to FLACC scale and sex of experimental group $(\mathrm{p}>0.05)\left(\chi^{2}=3.826, \mathrm{df}=2 \mathrm{p}=0.148\right)$

3 . There was no association found between pain score according to FLACC scale and previous experience of cannulation of experimental group $(\mathrm{p}>0.05)\left(\chi^{2}=0.024, \mathrm{df}=2\right.$, $\mathrm{p}=0.988)$.

4. That there was no association found between pain score according to FLACC scale and number of cannulation in the present hospitalization of experimental group $(\mathrm{p}>0.05)\left(\chi^{2}\right.$ $=2.806$, $\mathrm{df}=2, \mathrm{p}=0.246$ ).

5. There was no association found between pain score according to Wong Baker Faces Pain Rating scale and age of experimental group $(p>0.05)\left(\chi^{2}=4.786, d f=6, p=0.572\right)$.

6 . There was no association found between pain score according to Wong Baker Faces Pain Rating scale and sex of experimental group $(p>0.05)\left(\chi^{2}=4.484, d f=3, p=0.214\right)$.

7. There was no association found between pain score according to Wong Baker Faces Pain Rating scale and previous experience of cannulation of experimental group $(\mathrm{p}>0.05) \cdot\left(\chi^{2}=2.420, \mathrm{df}=3, \mathrm{p}=0.490\right)$.

8. There was no association found between pain score according to Wong Baker Faces Pain Rating scale and number of cannulation in the present hospitalization of experimental group $(p>0.05)\left(\chi^{2}=1.092, d f=3, p=0.779\right)$.

\section{Discussion}

Pain management is the alleviation of pain or a reduction in pain to a level of comfort that is acceptable to the client. It includes two basic types of nursing interventions: pharmacologic and non-pharmacologic methods [7]. The children need comforting activities to enhance pain management through clinical competence, distraction, participation and security [8].

In 2006, Tufekci et al conducted a study titled "Turkish children loved distraction: using kaleidoscope to reduce perceived pain during venipuncture" to assess the effect of distraction (looking through kaleidoscopes) to reduce perceived pain, during venipuncture in healthy school-age children. It was proved that pain levels of the children according to both scales - Wong Baker Faces Pain rating scale and visual analog scale, in intervention group were lower than those of control group. It was detected that the distraction made with kaleidoscope effectively reduced the pain related to venipuncture in healthy school children. Besides, the researchers suggested that distraction with kaleidoscope is a method, which the nurse will be able to use for venipuncture to obtain optimal pain control. The study was conducted in 206 school age children [3].

Vessey et al (1994) proved the use of kaleidoscope in distracting the children aged 3-11 years during venepuncture in reducing pain. The study was conducted in 100 children who were undergoing venipuncture [5].

In the present study also, it was proved that kaleidoscope was effective in managing pain in children during intravenous cannulation. The pain scores of experimental group assessed by two pain scales - FLACC and Wong Baker Faces Pain Rating scales, were comparatively lower than that of control group.

Fillingim R. B. et al (2009), in their study titled "Sex, gender and pain: A review of recent clinical and experimental findings", reported that sex difference in pain perception emerge or become larger around puberty [6].

Likewise, in this study also, it was found that there was no association between sex of the sample and their pain scores.

\section{Conclusion}

Based on the above mentioned results of the study it is evident that Kaleidoscope was effective in managing pain in children aged 4-6 years, during intravenous cannulation. Hence it is advisable to use different methods of distractions while doing the painful procedure. This helps not only the nurse to the ease the procedure but also it gives relief to the children by distracting their attention.

Moreover from the findings it's also clear that there was no association found between pain scores and any of the selected demographic variables like age, sex and the previous experience of cannulation.

\section{References}

[1] Srouji R. Ratnapalan S. \& Schneeweiss S. Pain in children: Assessment and nonpharmacological management. International Journal of Pediatrics. 2010, (2010) 10-21

[2] Potter P. A. \& Perry A. G. Fundamentals of nursing, Missouri: Mosby, 2015. P. 1228-1270.

[3] Tufekci et al. Turkish children loved distraction: using kaleidoscope to reduce perceived pain during venepuncture. Journal of Clinical Nursing, 2006, (18) 2180-2186.

[4] Hockenberry M. J. Wilson D. Essentials of Pediatric Nursing, Missouri: Mosby. 2009. p.159-191.

[5] Vessey et al. Cognitive behavior therapy for children and families. 2005, p.429.

[6] Fillingim R. B. et al. Sex, gender and pain: A review of recent clinical and experimental findings. Journal of pain, 2009, (10) 447-485.

[7] Kozier B et al. Fundamentals of nursing - Concepts, process \& practices, Singapore: Pearson, 2015. P.1132-1166.

[8] Nilsson S. et al. Children's experiences of procedural pain management in conjunction with trauma wound dressing. Journal of advanced nursing. 2011, (67) 1449-1457.

[9] Himali Raj Prajapati. A Study to Assess the Effectiveness of Kaleidoscope in Reducing Physical Stress during Venipuncture Procedure among Hospitalized Pre-School Children at Selected Hospital of Ahmadabad City, Gujarat State. Int. J. Nur. Edu. and Research. 2018; 6(1):44-46. 
[10] Unknown author. A study to assess the effectiveness of kaleidoscope on pain and behavioral responses among children (4-10years) during iv cannulation in selected hospital, coimbatore. October 2014. repositorytnmgrmu.ac.in/3145/1/3002154301216104Kallu\%20Das.pdf
[11] Becky Hix. Using distraction to reduce vene puncture pain and distress in school aged children: the role of the parents', nurses'and the child's voice: a review of the literature. 2014 https://pdfs.semanticscholar.org/ece8/1c85e70aacba9308ffe6a 1a0027bb7f68ab7.pdf 